\title{
Use of extracorporeal membrane oxygenation in patients with acute high-risk pulmonary embolism: a case series with literature review
}

\author{
You Na Oh${ }^{1}$, Dong Kyu Oh${ }^{1}$, Younsuck Koh¹, Chae-Man Lim', Jin-Won Huh', Jae Seung Lee', \\ Sung-Ho Jung ${ }^{2}$, Pil-Je Kang' ${ }^{2}$ Sang-Bum Hong ${ }^{1}$ \\ Departments of ${ }^{1}$ Pulmonary and Critical Care Medicine and ${ }^{2}$ Thoracic and Cardiovascular Surgery, Asan Medical Center, University of Ulsan College of \\ Medicine, Seoul, Korea
}

Background: Although extracorporeal membrane oxygenation (ECMO) has been used for the treatment of acute high-risk pulmonary embolism (PE), there are limited reports which focus on this approach. Herein, we described our experience with ECMO in patients with acute high-risk PE.

Methods: We retrospectively reviewed medical records of patients diagnosed with acute highrisk PE and treated with ECMO between January 2014 and December 2018.

Results: Among 16 patients included, median age was 51 years (interquartile range [IOR], 38 to 71 years) and six (37.5\%) were male. Cardiac arrest was occurred in $12(75.0 \%)$ including two cases of out-of-hospital arrest. All patients underwent veno-arterial ECMO and median ECMO duration was 1.5 days (IQR, 0.0 to 4.5 days). Systemic thrombolysis and surgical embolectomy were performed in seven $(43.8 \%)$ and nine $(56.3 \%)$ patients, respectively including three patients $(18.8 \%)$ received both treatments. Overall 30 -day mortality rate was $43.8 \%$ (95\% confidence interval, $23.1 \%$ to $66.8 \%$ ) and 30-day mortality rates according to the treatment groups were ECMO alone $(33.3 \%, n=3)$, ECMO with thrombolysis $(50.0 \%, n=4)$ and ECMO with embolectomy $(44.4 \%, n=9)$.

Conclusions: Despite the vigorous treatment efforts, patients with acute high-risk PE were related to substantial morbidity and mortality. We report our experience of ECMO as rescue therapy for refractory shock or cardiac arrest in patients with PE.

Key Words: embolectomy; extracorporeal membrane oxygenation; pulmonary embolism; shock; thrombolytic therapy; treatment outcome

\section{INTRODUCTION}

Acute pulmonary embolism (PE) is the most serious clinical presentation of venous thromboembolism which causes obstructive shock and hemodynamic instability [1-3]. It is stratified based on the early PE-related mortality risk and the high-risk group is defined as the patients who have shock or systemic hypotension [2,3]. Despite the expedited lifesaving treatments such as systemic thrombolysis and surgical embolectomy, unfortunately, acute highrisk PE is associated with significant morbidity and mortality [2-5].

\section{Original Article}

Received: April 10, 2019

Revised: May 16, 2019

Accepted: May 18, 2019

Corresponding author

Sang-Bum Hong

Department of Pulmonary and

Critical Care Medicine, Asan Medical

Center, University of Ulsan College of Medicine, 88 Olympic-ro 43-gil,

Songpa-gu, Seoul 05505, Korea

Tel: +82-2-3010-3893

Fax: +82-2-3010-6968

E-mail: hongsangbum@gmail.com

Copyright (c) 2019 The Korean Society of Critical Care Medicine

This is an Open Access article distributed under the terms of Creative Attributions Non-Commercial License (http://

creativecommons.org/li-censes/by-nc/4.0/) which permits unrestricted noncommercial use, distribution, and reproduction in any medium, provided the original work is properly cited. 
Veno-arterial extracorporeal membrane oxygenation (V-A ECMO) which bypasses both lungs and heart is a potentially lifesaving therapeutic option by providing hemodynamic and respiratory support in patients with acute high-risk PE [5-7]. It may serve as a bridge therapy to systemic thrombolysis or surgical embolectomy or as a stand-alone therapy without primary reperfusion, which supports patients mechanically while waiting for the activation of innate physiologic thrombolysis [5-7]. To date, however, there have been limited reports describing this approach and the literatures still have not provided sufficient evidence whether the ECMO is an effective therapeutic option for the acute high-risk PE $[2,3,8]$. In this context, we described our experience with ECMO in patients with acute high-risk PE and reviewed the related literatures.

\section{MATERIALS AND METHODS}

\section{Study Design and Data Collection}

The study was performed at Asan Medical Center, a tertiary referral teaching hospital in Seoul, Korea, where the annual ECMO volume is $>200$ cases. The data were retrospectively collected from adult patients ( $\geq 19$ years old) who were diagnosed with acute high-risk PE and treated with ECMO between January 2014 and December 2018. According to the latest American Heart Association and European Society of Cardiology guidelines, the acute high-risk PE was defined as suspected or confirmed acute PE in the presence of shock or persistent arterial hypotension and patients whose systolic blood pressure $<90 \mathrm{mmHg}$ or drop by $\geq 40 \mathrm{mmHg}$ for $>15$ minutes were regarded as having a persistent arterial hypotension [2,3]. In our institution, ECMO was performed by a specialized ECMO team which consists of cardiothoracic surgeons and perfusionists. Because we included patients with acute high-risk PE who require urgent cardiopulmonary and respiratory support, peripherally inserted V-A ECMO of which drainage cannulation at femoral vein and return cannulation at femoral artery was primarily considered as an initial configuration in all cases.

Data on baseline characteristics were retrieved at the time of diagnosis, which include age, gender, body mass index, smoking history, comorbidities, predisposing factors for venous thromboembolism, presenting symptoms and signs, and diagnostic modalities. We also investigated major procedures performed for the treatment of acute high-risk PE including systemic thrombolysis and surgical embolectomy. Clinical outcomes were compared in terms of 30-day mortality rate and ECMO weaning rate according to the treatment modalities; thrombolysis group vs. nonthrombolysis group and embolec-

\section{KEY MESSAGES}

- Overall 30-day mortality rate was $43.8 \%$ in patients with acute high-risk pulmonary embolism who required hemodynamic and respiratory support with extracorporeal membrane oxygenation (ECMO).

- We divided three group of patients such as ECMO alone, ECMO with thrombolysis, and ECMO with embolectomy.

- ECMO was treated in conjunction with either systemic thrombolysis or surgical embolectomy in patients with acute high-risk pulmonary embolism.

tomy group vs. non-embolectomy group. Complications were investigated and compared according to the treatment groups as well.

The study was performed according to the Helsinki Declaration and approved by the Institutional Review Board of Asan Medical Center (IRB No. 2019-0359). The informed consent was waived because of the retrospective nature of the study.

\section{Statistical Analysis}

Continuous variables were summarized using means with standard deviations and categorical variables were summarized using frequencies with proportions. Kaplan-Meier curves were plotted for the survival analyses. Statistical analyses were performed using IBM SPSS ver. 21.0 (IBM Corp., Armonk, NY, USA).

\section{RESULTS}

\section{Baseline Characteristics}

We identified 16 patients with acute high-risk PE who were treated with ECMO between January 2014 and December 2018. Table 1 describes the baseline characteristics of included patients. The median age was 51 years (interquartile range [IQR], 38 to 71 years) and six patients $(37.5 \%)$ were male. The most common predisposing factor for venous thromboembolism was recent hospitalization $(n=7,43.7 \%)$ followed by active cancer $(n=6,37.5 \%)$ and recent invasive vascular procedures $(n=5,31.2 \%)$. Four patients $(25.0 \%)$ were pregnant at the time of diagnosis and previous history of venous thromboembolism was reported in one patient $(6.2 \%)$.

\section{Initial Presentation and Diagnosis}

Initial symptoms and signs and the diagnostic modalities are described in Table 2. The most common initial presentation was dyspnea $(n=6,37.5 \%)$ followed by hemodynamic collapse 
Table 1. Baseline characteristics of the included patients

\begin{tabular}{lc}
\hline Variable & Value $(\mathrm{n}=16)$ \\
\hline Age $(\mathrm{yr})$ & $51(38-71)$ \\
Male & $6(37.5)$ \\
Body mass index $\left(\mathrm{kg} / \mathrm{m}^{2}\right)$ & $25.0(22.6-32.7)$ \\
Smoking & $2(12.5)$ \\
Comorbidity & \\
Diabetes & $4(25.0)$ \\
Hypertension & $4(25.0)$ \\
Chronic kidney disease & 0 \\
Predisposing factor & \\
Recent hospitalization & $7(43.7)$ \\
Active cancer & $6(37.5)$ \\
Recent invasive procedure & $5(31.2)$ \\
Pregnancy & $4(25.0)$ \\
Immobilization & $3(18.8)$ \\
History of venous thromboembolism & $1(6.2)$ \\
Recent trauma & $1(6.2)$ \\
Hormone therapy & 0 \\
Thrombophilia & 0 \\
\hline
\end{tabular}

Values are presented as median (interquartile range) or number (\%).

$(\mathrm{n}=4,25.0 \%)$. Cardiac arrest was reported in $12(75.0 \%)$ including two cases occurred at out-of-hospital in Figure 1. Among 13 patients who performed computed tomography (CT) angiography, right ventricle strain pattern which was defined as the ratio of right ventricle and left ventricle ratio $>1$ was observed in 12 patients (92.3\%). The diagnosis was confirmed without CT angiography in three patients (18.8\%). Two patients were suspected of acute PE on echocardiography and the diagnosis was confirmed during surgical embolectomy. A patient was presented with cardiac arrest and initially suspected of acute coronary syndrome. He was transferred for coronary angiography, but the there was no abnormality in coronary arteries and acute PE was diagnosed during pulmonary angiography. Echocardiography was performed in all patients of which 15 (93.8\%) showed right ventricular dilatation and dysfunction.

\section{Therapeutic Procedures}

Procedures performed for the treatment of acute high-risk PE are described in Table 3 . All patients required mechanical support with V-A ECMO and the median ECMO duration was 1.5 days (IQR, 0.0 to 4.5 days). Among 16 patients included, three (18.8\%) were treated with ECMO alone and 13 (81.3\%) were bridged to one or more primary reperfusion therapies such as
Table 2. Initial presentation and diagnosis of acute high-risk pulmonary embolism

\begin{tabular}{lc}
\hline Variable & Value $(\mathrm{n}=16)$ \\
\hline Initial symptom & \\
Dyspnea & $6(37.5)$ \\
Hemodynamic collapse & $4(25.0)$ \\
Syncope & $3(18.8)$ \\
Altered mentality & $2(12.5)$ \\
Leg pain or swelling & $1(6.2)$ \\
Cardiac arrest & $12(75.0)$ \\
In-hospital & $10(83.3)$ \\
Out-of-hospital & $2(16.7)$ \\
CT angiography & $13(81.2)$ \\
Saddle pulmonary embolism on CT & $9(69.2)$ \\
RV strain on CT & $12(92.3)$ \\
Echocardiography & $16(100.0)$ \\
RV dysfunction & $15(93.8)$ \\
RV dilatation & $15(93.8)$ \\
LV ejection fraction (\%) & $21(9-53)$ \\
Laboratory finding & \\
D-dimer ( $\mu$ g/ml) & $24.1(6.7-35.2)$ \\
CK-MB (ng/ml) & $4.0(1.8-9.2)$ \\
Troponin I (pg/ml) & $0.3(0.1-1.2)$ \\
BNP (pg/ml) & $171(65-347)$ \\
Lactate (mmol/L) & $9.4(4.9-13.3)$ \\
\hline & \\
\hline
\end{tabular}

Values are presented as number (\%) or median (interquartile range). CT: computed tomography; RV: right ventricle; LV: left ventricle; CKMB: creatine kinase-muscle/brain; BNP: brain natriuretic peptide.

systemic thrombolysis and surgical embolectomy. Systemic thrombolysis and surgical embolectomy were performed in seven patients (43.8\%) and nine patients (56.3\%), respectively including three patients (18.8\%) who received both treatments; in these patients, systemic thrombolysis was followed by surgical embolectomy because of persistent shock and systemic hypotension. No catheter-based thrombolysis was performed because it was not available in our hospital.

\section{Clinical Outcomes and Complications}

The overall 30-day mortality was occurred in seven patients (43.8\%) and the ECMO was successfully weaned in 10 patients (62.5\%) (Table 4, Figure 2). The most common complication was bleeding $(n=9,56.3 \%)$ which requires transfusion of $\geq 2$ packed red blood cells. Cerebrovascular accident and arterial ischemia were reported in one patient (6.2\%), respectively, and both were assessed to be associated with ECMO support. 


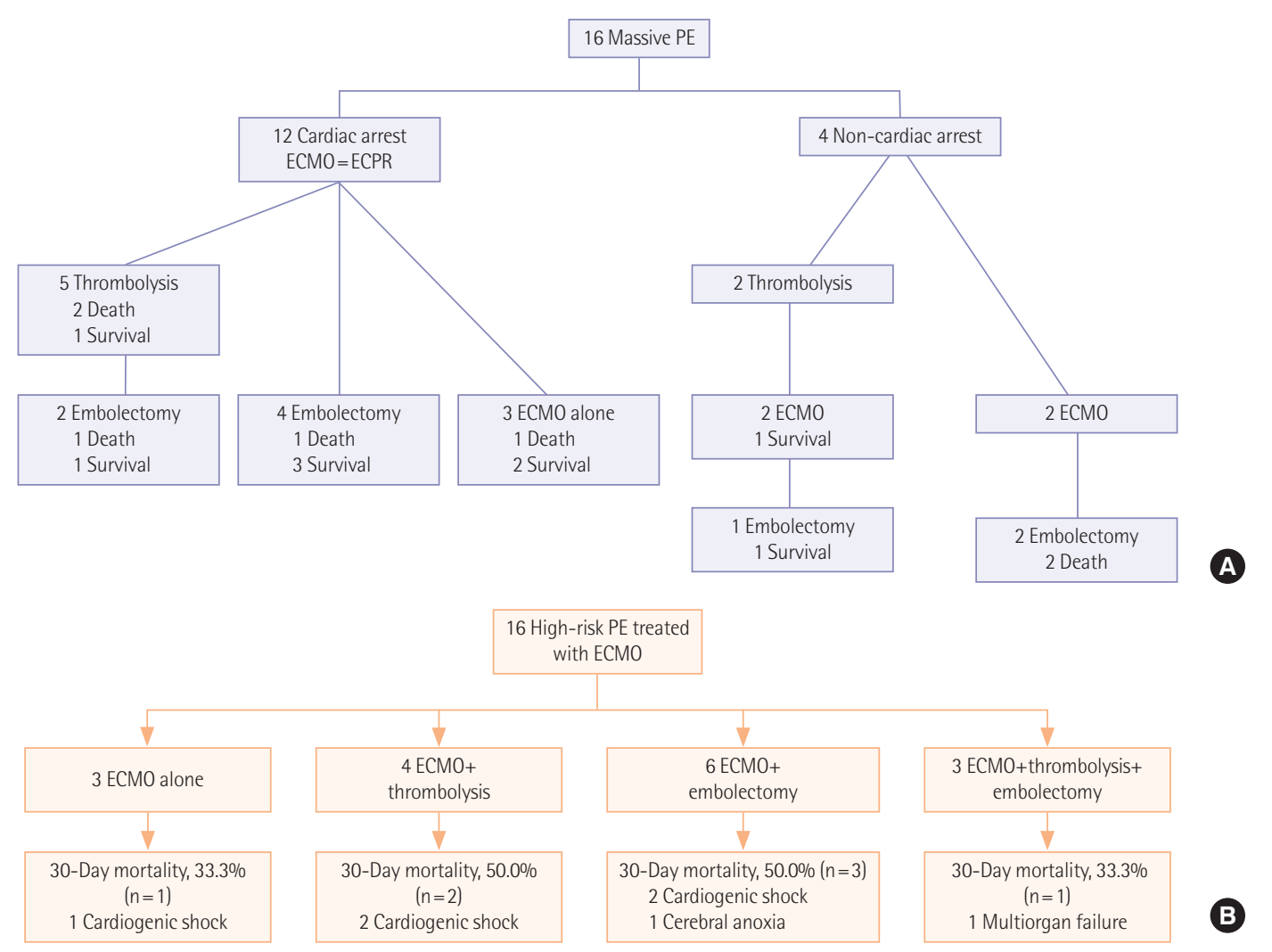

Figure 1. (A) A timeline of treatment strategies and 30-day mortalities. (B) A flowchart of treatment strategies and 30-day mortalities with causes of death. PE: pulmonary embolism; ECMO: extracorporeal membrane oxygenation; ECPR: extracorporeal cardiopulmonary resuscitation.

Table 3. Treatment of acute high-risk pulmonary embolism

\begin{tabular}{lc}
\hline Variable & Value $(n=6)$ \\
\hline ECMO & $16(100.0)$ \\
Duration (day) & $1.5(0.0-4.5)$ \\
V-A ECMO & $16(100.0)$ \\
Central V-A ECMO & $2(12.5)$ \\
IVC filter & $2(12.5)$ \\
Treatment strategy & \\
ECMO alone & $3(18.8)$ \\
ECMO+systemic thrombolysis & $4(25.0)$ \\
ECMO+surgical embolectomy & $6(37.5)$ \\
ECMO+systemic thrombolysis+surgical embolectomy & $3(18.8)$ \\
\hline
\end{tabular}

Values are presented as number (\%) or median (interquartile range). ECMO: extracorporeal membrane oxygenation; V-A: veno-arterial; IVC: inferior vena cava.

In Figure 1, we described 30-day mortalities and causes of death according to the treatment strategies. Despite the vigorous treatment efforts, substantial mortalities were reported in each treatment group (33.3\%-50.0\%). The most common cause of death was cardiogenic shock $(n=5,71.4 \%)$. Cerebral anoxia
Table 4. Clinical outcomes and complications according to treatment strategies

\begin{tabular}{lccc}
\hline Variable & $\begin{array}{c}\text { ECM0 alone } \\
(n=3)\end{array}$ & $\begin{array}{c}\text { ECM0 with } \\
\text { thrombolysis } \\
(n=4)\end{array}$ & $\begin{array}{c}\text { ECM0 with } \\
\text { embolectomy } \\
(n=9)\end{array}$ \\
\hline 30-Day mortality & $1(33.3)$ & $2(50.0)$ & $4(44.4)$ \\
ECM0 weaning & $2(66.7)$ & $2(50.0)$ & $6(66.7)$ \\
Bleeding & $1(33.3)$ & $1(25.0)$ & $7(77.8)$ \\
Cerebrovascular event & 0 & 0 & $1(11.1)$ \\
Wound infection & 0 & $1(25.0)$ & $1(11.1)$ \\
Arterial ischemia & 0 & 0 & $1(11.1)$ \\
Recurrent DVT & 0 & 0 & $1(11.1)$ \\
CTEPH & 0 & 0 & 0 \\
\hline
\end{tabular}

Values are presented as number (\%).

ECMO: extracorporeal membrane oxygenation; DVT: deep vein thrombosis; CTEPH: chronic thromboembolic pulmonary hypertension.

and multiorgan failure were reported in a case (6.3\%), respectively. We also compared clinical outcomes and complications according to the treatment groups; 30-day mortality rate was $43.8 \%$ (95\% confidence interval, $23.1 \%$ to $66.8 \%$ ) and 30 -day mortality rates according to the treatment groups was ECMO 


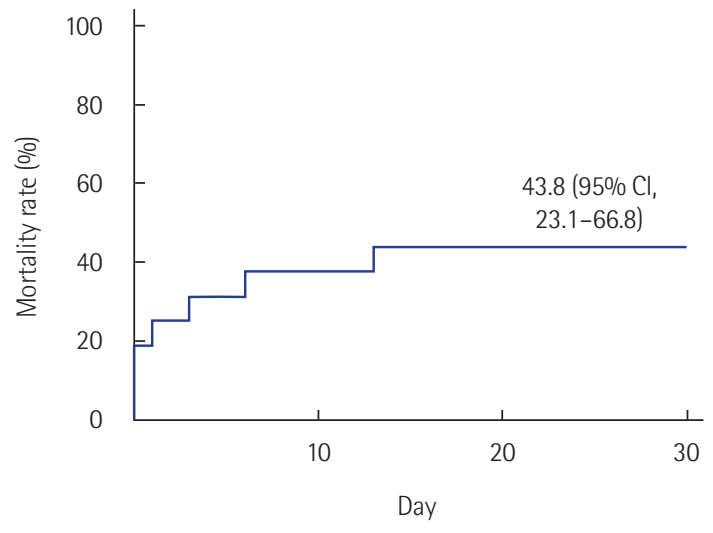

Figure 2. Thirty-day mortality. Cl: confidence interval.

alone $(n=3,33.3 \%)$, ECMO with thrombolysis $(n=4,50.0 \%)$ and ECMO with embolectomy $(n=9,44.4 \%)$ (Table 4).

We showed outcome of patients by initial cardiac arrest or not (Table 5). Besides four peripartum patients were included. After C-sec, PE developed three patients and a patient after $\mathrm{D} \& \mathrm{C}$ due to ectopic pregnancy. We did all four patients ECPR, one patient died during extracorporeal cardiopulmonary resuscitation, and three patients survived. Among three patients, one patient received thrombolysis, one for embolectomy, and one for ECMO alone.

\section{DISCUSSION}

The management of acute high-risk PE requires multifaceted approaches including hemodynamic and respiratory support in addition to anticoagulation and primary reperfusion therapy $[2,3]$. Several approaches have been suggested to provide hemodynamic and respiratory support, which includes fluid challenge, use of vasopressors and inotropes, inhalation of nitric oxide, and mechanical ventilation with minimal positive end expiratory pressure. However, none has been proved to provide sufficient hemodynamic and respiratory support in patients with acute high-risk PE [2,3,9-12]. Recently, there is an increasing attention to the utilization of ECMO in the treatment of acute PE which causes hemodynamic instability [5]. But the notion is only supported by occasional case reports and patient series and the literatures still have not provided sufficient evidence whether the ECMO is an effective option for the treatment of acute high-risk PE [2,3,5-8,13-15].

In this consecutive case series, we presented our data on 16 patients with confirmed acute high-risk $\mathrm{PE}$ who required both hemodynamic and respiratory support with V-A ECMO. Systemic thrombolysis and surgical embolectomy were performed in seven and nine patients, respectively including three patients
Table 5. ECMO outcomes and ICU events according to CPR

\begin{tabular}{lcc}
\hline Outcome/event & Non-CPR $(n=4)$ & CPR $(n=12)$ \\
\hline 30-Day mortality & $2(50.0)$ & $5(41.7)$ \\
In-hospital mortality & $2(50.0)$ & $7(58.3)$ \\
Total mortality & $2(50.0)$ & $7(58.3)$ \\
ECMO duration (day) & $5.2 \pm 5.3$ & $3.0 \pm 4.6$ \\
ICU LOS (day) & $8.5 \pm 5.3$ & $12.3 \pm 15.2$ \\
Hospital LOS (day) & $25.8 \pm 19.9$ & $46.7 \pm 56.8$ \\
Thrombolysis & $2(50.0)$ & $5(41.7)$ \\
Embolectomy & $3(75.0)$ & $6(50.0)$ \\
Acute kidney injury requiring RRT & $2(50.0)$ & $7(58.3)$ \\
Cerebrovascular event & 0 & $1(8.3)$ \\
Wound infection & 0 & $2(16.7)$ \\
Artery ischemia & 0 & $1(8.3)$ \\
Recurrent DVT & $1(25.0)$ & 0 \\
CTEPH & 0 & 0
\end{tabular}

Values are presented as number (\%) or mean \pm standard deviation. ECMO: extracorporeal membrane oxygenation; ICU: intensive care unit; CPR: cardiopulmonary resuscitation; LOS: length of stay; RRT: renal replacement therapy; DVT: deep vein thrombosis; CTEPH: chronic thromboembolic pulmonary hypertension.

who received both treatments. Despite the vigorous therapeutic efforts, patients with acute high-risk PE had significant morbidity and mortality and seven patients (43.8\%) died within 30 days from the initial diagnosis. The overall 30-day mortality rate, $43.8 \%$, reported in our study seems to be high, but is similar to the results reported in other studies which support the potential benefit of ECMO in patients with acute high-risk PE $[6,7,13-15]$. Moreover, in the present study, three-quarters of patients were initially presented with cardiac arrest which is a well-known risk factor for mortality even when full standard resuscitative efforts are employed $[5,16]$. Considering the higher mortality rate in patients with acute PE who developed cardiac arrest, which ranges from $52 \%$ to $65 \%$ [17], further study is needed for the potential benefit of ECMO in patients with acute high-risk $\mathrm{PE}$.

Historically surgical embolectomy was the treatment of choice for acute PE which causes shock or systemic hypotension, but less invasive procedures such as systemic thrombolysis have been introduced during last decades $[5,18,19]$. Currently, both treatments are recommended by the latest clinical guidelines and, in a recent systematic review, there is no evidence that one is superior to the other treatment particularly in patients treated with ECMO $[2,3,5]$. In the present study, 30-day mortality rates and ECMO weaning rates were similar among treatments. 
Treatment with ECMO alone also may be considered in some patients with acute high-risk PE. It has been shown that the recovery of right ventricle and hemodynamic improvement may be achieved within 5 days from the ECMO support by the activation of innate physiologic thrombolysis [17]. In our study, three patients were treated with ECMO alone without any primary reperfusion therapy. Although one of them, who was initially presented with out-of-hospital cardiac arrest and required > 30 minutes until the initiation of ECMO support, died within a few hours after admission, the remaining are survived without other invasive procedures. This approach may be beneficial by avoiding potential risks of primary reperfusion therapy such as major bleeding and surgical site infection, but currently there are limited data regarding who will be successfully bridged to recovery or who will require primary reperfusion therapy. Further studies are required to assess the clinical outcomes and prognostic factors in patients with acute high-risk PE, treated with ECMO support alone.

There are several limitations in our study. The study was retrospectively performed in a single center and may be prone to bias. The relatively small number of patients included in the study also limit the generalization of results. Nonetheless, it should be noted that this is an area where the randomized controlled trials are virtually impossible and the results from cases reports and patient series are important to guide clinical practices.

In conclusion, despite the vigorous treatment efforts, patients with acute high-risk PE were related to substantial morbidity and mortality. We report our experience of ECMO as rescue therapy for refractory shock or cardiac arrest in patients with PE. Further investigations are required in larger samples to confirm our findings.

\section{CONFLICT OF INTEREST}

No potential conflict of interest relevant to this article was reported.

\section{ORCID}

$\begin{array}{ll}\text { You Na Oh } & \text { https://orcid.org/0000-0002-2647-8403 } \\ \text { Dong Kyu Oh } & \text { https://orcid.org/0000-0002-7511-9634 } \\ \text { Younsuck Koh } & \text { https://orcid.org/0000-0001-5066-2027 } \\ \text { Chae-Man Lim } & \text { https://orcid.org/0000-0001-5400-6588 } \\ \text { Jin-Won Huh } & \text { https://orcid.org/0000-0002-3449-0461 } \\ \text { Jae Seung Lee } & \text { https://orcid.org/0000-0003-4130-1486 } \\ \text { Sung-Ho Jung } & \text { https://orcid.org/0000-0002-3699-0312 }\end{array}$

Pil-Je Kang https://orcid.org/0000-0002-7587-0911

Sang-Bum Hong https://orcid.org/0000-0003-2737-7695

\section{AUTHOR CONTRIBUTIONS}

Conceptualization: SBH. Data curation: YNO, DKO, JSL, SHJ, PJK. Formal analysis: YNO, DKO, SBH. Methodology: YNO, DKO, YK, CML, JWH, JSL, SBH. Project administration: YNO, DKO, SBH. Visualization: YNO, DKO. Writing - original draft: YNO, DKO. Writing - review \& editing: all authors.

\section{REFERENCES}

1. Aissaoui N, Konstantinides S, Meyer G. What's new in severe pulmonary embolism? Intensive Care Med 2019;45:75-7.

2. Konstantinides SV, Torbicki A, Agnelli G, Danchin N, Fitzmaurice $\mathrm{D}$, Galiè N, et al. 2014 ESC guidelines on the diagnosis and management of acute pulmonary embolism. Eur Heart J 2014;35:3033-69.

3. Jaff MR, McMurtry MS, Archer SL, Cushman M, Goldenberg $\mathrm{N}$, Goldhaber SZ, et al. Management of massive and submassive pulmonary embolism, iliofemoral deep vein thrombosis, and chronic thromboembolic pulmonary hypertension: a scientific statement from the American Heart Association. Circulation 2011;123:1788-830.

4. Logan JK, Pantle H, Huiras P, Bessman E, Bright L. Evidencebased diagnosis and thrombolytic treatment of cardiac arrest or periarrest due to suspected pulmonary embolism. Am J Emerg Med 2014;32:789-96.

5. Yusuff HO, Zochios V, Vuylsteke A. Extracorporeal membrane oxygenation in acute massive pulmonary embolism: a systematic review. Perfusion 2015;30:611-6.

6. Corsi F, Lebreton G, Bréchot N, Hekimian G, Nieszkowska A, Trouillet JL, et al. Life-threatening massive pulmonary embolism rescued by venoarterial-extracorporeal membrane oxygenation. Crit Care 2017;21:76.

7. Meneveau N, Guillon B, Planquette B, Piton G, Kimmoun A, Gaide-Chevronnay L, et al. Outcomes after extracorporeal membrane oxygenation for the treatment of high-risk pulmonary embolism: a multicentre series of 52 cases. Eur Heart J 2018;39:4196-204.

8. Galiè N, Palazzini M, Manes A. Extracorporeal cardiopulmonary support in acute high-risk pulmonary embolism: still waiting for solid evidence. Eur Heart J 2018;39:4205-7.

9. Manier G, Castaing Y. Influence of cardiac output on oxygen exchange in acute pulmonary embolism. Am Rev Respir Dis 1992;145:130-6. 
10. Mercat A, Diehl JL, Meyer G, Teboul JL, Sors H. Hemodynamic effects of fluid loading in acute massive pulmonary embolism. Crit Care Med 1999;27:540-4.

11. Capellier G, Jacques T, Balvay P, Blasco G, Belle E, Barale F. Inhaled nitric oxide in patients with pulmonary embolism. Intensive Care Med 1997;23:1089-92.

12. Szold O, Khoury W, Biderman P, Klausner JM, Halpern P, Weinbroum AA. Inhaled nitric oxide improves pulmonary functions following massive pulmonary embolism: a report of four patients and review of the literature. Lung 2006;184:1-5.

13. Dolmatova EV, Moazzami K, Cocke TP, Elmann E, Vaidya P, $\mathrm{Ng} \mathrm{AF}$, et al. Extracorporeal membrane oxygenation in massive pulmonary embolism. Heart Lung 2017;46:106-9.

14. Kjaergaard B, Kristensen JH, Sindby JE, de Neergaard S, Rasmussen BS. Extracorporeal membrane oxygenation in lifethreatening massive pulmonary embolism. Perfusion 2019: 267659119830014.

15. George B, Parazino M, Omar HR, Davis G, Guglin M, Gurley J, et al. A retrospective comparison of survivors and non-survivors of massive pulmonary embolism receiving veno-arterial extracorporeal membrane oxygenation support. Resuscitation 2018;122:1-5.

16. Sakuma M, Nakamura M, Yamada N, Nakano T, Shirato K. Percutaneous cardiopulmonary support for the treatment of acute pulmonary embolism: summarized review of the literature in Japan including our own experience. Ann Vasc Dis 2009;2:7-16.

17. Maggio P, Hemmila M, Haft J, Bartlett R. Extracorporeal life support for massive pulmonary embolism. J Trauma 2007; 62:570-6.

18. Leacche M, Unic D, Goldhaber SZ, Rawn JD, Aranki SF, Couper GS, et al. Modern surgical treatment of massive pulmonary embolism: results in 47 consecutive patients after rapid diagnosis and aggressive surgical approach. J Thorac Cardiovasc Surg 2005;129:1018-23.

19. Wan S, Quinlan DJ, Agnelli G, Eikelboom JW. Thrombolysis compared with heparin for the initial treatment of pulmonary embolism: a meta-analysis of the randomized controlled trials. Circulation 2004;110:744-9. 